\title{
Nutrition knowledge related to breakfast skipping among Japanese adults aged 18-64 years: a cross-sectional study
}

\author{
Mai Matsumoto ${ }^{1,2}$, Naomi Ishige ${ }^{1}$, Azusa Sakamoto ${ }^{1}$, Aki Saito ${ }^{2}$ and Shinji lkemoto ${ }^{1, *}$ \\ ${ }^{1}$ Department of Human Nutrition, Seitoku University, Iwase 550, Matsudo-shi, Chiba 271-8555, Japan: \\ ${ }^{2}$ Department of Nutritional Epidemiology and Shokuiku, National Institute of Biomedical Innovation, Health and \\ Nutrition, Tokyo, Japan
}

Submitted 17 April 2018: Final revision received 5 September 2018: Accepted 1 0ctober 2018: First published online 19 November 2018

\begin{abstract}
Objective: Breakfast skipping is regarded as a public health issue among adults worldwide. Nutrition knowledge has been reported to be one of predictors of dietary behaviour. Therefore, the aim of the present study was to examine the association between nutrition knowledge and breakfast skipping.

Design: Data regarding nutrition knowledge were obtained by using a validated, self-administered general nutrition knowledge questionnaire for Japanese adults (JGNKQ). Participants were classified into three nutrition knowledge level groups according to total JGNKQ score: Low, Middle and High. In addition, participants reported the frequency of meal consumption per week and rated the difficulty in finding time to eat breakfast, lunch and dinner in the lifestyle questionnaire. The differences in frequency of breakfast, lunch and dinner consumption among Low, Middle and High nutrition knowledge groups were determined by using ANCOVA adjusted for potential confounding factors.

Setting: Kanto region, Japan.

Participants: Japanese adults aged 18-64 years ( $n$ 1165, 57.3\% women).

Results: Mean age of the participants was 43.8 (SD 8.9) years. There were no significant differences found in the proportion of respondents reporting difficulty in finding time to eat each meal among the three groups. However, the frequency of breakfast consumption was significantly different among Low, Middle and High groups, while lunch and dinner frequency did not differ among the three groups. Conclusions: The present study suggests that nutrition knowledge level is related to breakfast skipping among Japanese adults.
\end{abstract}

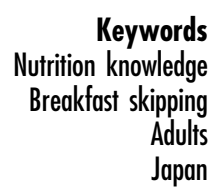

Eating behaviours can be influenced by various factors. Nutrition knowledge has been reported to affect eating behaviours of adults ${ }^{(1,2)}$. Several studies have reported that people with higher nutrition knowledge level practise better dietary behaviours such as higher consumption of vegetables, fruits, fibre and micronutrients and less fat than those with lower nutrition knowledge level ${ }^{(2-6)}$. Additionally, one study has suggested that nutrition knowledge is one of the factors necessary to improve dietary behaviours $^{(7)}$. For example, it has been reported that adults with knowledge about dietary fat and cholesterol effectively improved those nutrients' intake ${ }^{(8)}$. Thus, nutrition knowledge may lead to appropriate dietary behaviours.

Eating breakfast has been reported as a predictor of healthy eating behaviours such as high intake of dietary fibre, thiamin or folate and low intake of fat in previous cross-sectional and intervention studies ${ }^{(9-11)}$. In addition, it has been reported that breakfast skipping affects individuals' health status. For example, breakfast skipping was reported to be associated with weight gain ${ }^{(12,13)}$ and increase of $\mathrm{BMI}^{(14,15)}$. Moreover, breakfast skipping was reported to be associated with higher prevalence of obesity $^{(13)}$, hypertension ${ }^{(16,17)}$, hyperlipidaemia ${ }^{(17,18)}$, insulin insensitivity and type 2 diabetes ${ }^{(13)}$, and $\mathrm{CVD}^{(17,19)}$. Thus, breakfast skipping is likely to lead to a worse health condition. The rate of breakfast skipping among Japanese adults has not decreased over the past 10 years ${ }^{(20)}$. Similarly, breakfast skipping has been reported as a public health issue all over the world including among $\mathrm{US}^{(21)}$ and UK adults ${ }^{(22)}$. Therefore, efforts to improve the behaviour of breakfast skipping are required after clarifying the factors related to breakfast skipping throughout the world.

Breakfast skipping may be improved by nutrition knowledge. However, there are only a few studies that have examined the relationship between breakfast skipping and nutrition knowledge. According to a previous 
study, it was indicated that people with lower nutrition knowledge level tended to skip breakfast ${ }^{(23)}$. However, that study examined a limited area of nutrition knowledge using a questionnaire that included only eight question items derived from the General Nutrition Knowledge Questionnaire (GNKQ) comprising 110 questions ${ }^{(24)}$. Thus, the relationship between breakfast skipping and nutrition knowledge has not been completely clarified yet. To investigate the association between breakfast skipping and nutrition knowledge, it is needed to study the breakfast skipping-nutrition knowledge relationship using an overall general nutrition knowledge questionnaire that has reasonable validity and reliability. Therefore, the aim of the present study was to evaluate the association of breakfast skipping and overall nutrition knowledge level.

\section{Methods}

\section{Procedure and study population}

Japanese adults aged 18-64 years were recruited from students' parents or acquaintances through seven kindergartens, one elementary school, two junior high schools, two high schools and one university in Tokyo, Chiba and Ibaraki prefectures, Kanto region, Japan, from January to March 2015. A set of two self-administered questionnaires (i.e. a Japanese general nutrition knowledge questionnaire (JGNKQ) and a lifestyle questionnaire) was distributed to a total of 5418 Japanese adults (2709 men and 2709 women) aged 18 to 64 years. The completed questionnaires which were collected by mail were examined by the research staff, and those with missing information were returned to the participants for completion. Both questionnaires were completed by 1281 Japanese adults (response rate: $23.6 \%$ ).

We excluded the following respondents: those who had missing data ( $n$ 98), those who reported age under 18 years or over 65 years $(n 7)$ and those who were receiving nutritional counselling by a doctor or registered dietitian ( $n$ 11). Thus, the final participant list consisted of 1165 Japanese adults. Written informed consent was obtained from all participants.

\section{Assessment of nutrition knowledge level}

Nutrition knowledge level was assessed using a previously validated Japanese general nutrition knowledge questionnaire (JGNKQ) ${ }^{(25)}$. Details of the JGNKQ's structure, validity and reliability have been published elsewhere ${ }^{(25)}$. Briefly, the JGNKQ is a self-administered ten-page questionnaire including five sections, i.e. 'Dietary recommendations' (section 1; nine items), 'Sources of nutrients' (section 2; ninety-six items), 'Choosing everyday foods' (section 3; five items), 'Diet-disease relationships' (section 4; twenty items) and 'Reading a food label' (section 5; seventeen items), with a total of 147 items.
The responses were converted to binary numbers, with 1 and 0 representing correct and incorrect answers, respectively. Therefore, the maximum score on the $147-$ item questionnaire was 147 points. A higher score reflects a higher knowledge level. In the current study, we classified participants into three nutrition knowledge level groups according to the JGNKQ total score: Low, Middle and High.

\section{Frequency of meals and difficulty of the behaviour having time to sit down and eat}

Participants reported difficulty of the behaviour having time to sit down and eat at breakfast, lunch and dinner and the frequency of breakfast, lunch and dinner per week in the lifestyle questionnaire, by asking, 'Is it usually difficult to sit down and eat for you at breakfast, lunch and dinner, respectively?' and 'How many times per week do you have breakfast, lunch and dinner, respectively? The definition of breakfast was the first meal of the day, eaten before or at the start of daily activities (e.g. errands, travel, work), within 2 h of waking, typically no later than 10.00 hours, and of energy level between 20 and $35 \%$ of total daily energy needs, which is defined by Timlin and Pereira ${ }^{(26)}$. Due to the lack of any standard definition of lunch and dinner time in the literature, we defined that lunch and dinner were the meals eaten from 12.00 to 14.00 hours and from 16.00 to 20.00 hours, respectively. Difficulty of the behaviour having time to sit down and eat was asked of participants using a 5-point Likert scale ('very difficult', 'difficult', 'in between', 'easy', 'very easy').

\section{Other variables}

In the lifestyle questionnaire, the participants reported their sex, age, educational background (junior high school or high school; junior college or vocational technical school; university or higher), annual household income (<2 million yen; 2-6 million yen; 6-10 million yen; >10 million yen), working status (full-time; part-time; housewife; student; others); marital status (yes; no) and number of children $(0 ; 1 ; 2 ; \geq 3)$.

\section{Statistical analysis}

All statistical analyses were performed using the statistical software package IBM SPSS Statistics version 22.0. All reported $P$ values are two-tailed, with $P<0.05$ considered statistically significant. The differences in characteristics between Low, Middle and High groups were compared using the $\chi^{2}$ test for categorical variables and ANOVA for continuous variables. The proportions of difficulty of the behaviour having time to sit down and eat were compared by the $\chi^{2}$ test among Low, Middle and High groups.

Additionally, multivariate-adjusted means with SE for meal frequency per week were calculated among Japanese adults categorized into Low, Middle and High groups, 
respectively. Potential confounding factors considered in the first analysis were sex, working status and number of children, which were found to be significantly different $(P<0.05)$ between groups categorized by nutrition knowledge level. Then, the confounding factors in the second analysis were the first model factors and age, which was reported as a factor affecting breakfast skipping among Japanese ${ }^{(15,27)}$. The adjusted differences in frequency of breakfast, lunch and dinner between Low, Middle and High groups were assessed by ANCOVA.

\section{Results}

Basic characteristics of participants are shown in Table 1. The proportion of women was $57.3 \%$, and the mean age of the study participants was $43 \cdot 8$ (SD 8.9 ) years. The main characteristics of participants were having a university degree ( $41.5 \%)$, having an annual income of 6-10 million yen ( $42 \cdot 1 \%)$ and being employed full-time (45.8\%). Additionally, the mean total nutrition knowledge score of all participants was 69.3 (SD 23.7). Significantly more female participants were categorized into the High nutrition knowledge group than the Low nutrition knowledge group $(P<0.001)$. Compared with participants in the High group, those in the Low group had a higher proportion of full-time workers and a lower proportion of housewives $(P<0 \cdot 001)$. Significantly more participants with a child were categorized into the High group than the Low and Middle groups $(P=0.002)$. There were no significant differences in age, education, annual household income and marital status among participants categorized in Low, Middle and High groups.

The proportions of difficulty of the behaviour having time to sit down and eat at breakfast, lunch and dinner in the Low, Middle and High groups are shown in Table 2. The percentage of those who answered 'very difficult' or 'difficult' to the question 'Is it usually difficult to sit down and eat for you at breakfast, lunch and dinner, respectively?' was less than 7,5 and $4 \%$, respectively, in all groups. Additionally, no significant differences were observed in the proportions of the difficulty of having time to sit down and eat at breakfast, lunch and dinner according to nutrition knowledge level.

Table 3 shows the frequency of breakfast, lunch and dinner per week among participants who were categorized in the Low, Middle and High groups. The mean number of days of eating breakfast was $5 \cdot 3$ (SE $0 \cdot 1$ ) in the Low group, $5 \cdot 6$ (se 0.1) in the Middle group and $5 \cdot 7$ (SE 0.1) in the High group. The mean number of days of lunch or dinner consumption was more than $6 \cdot 0$, and the number of days of lunch or dinner skipping was less than that of breakfast in all groups. The breakfast frequency was significantly different between Low, Middle and High groups after adjusting for sex (men or women), working status (full-time, part-time, housewife, student or other) and number of children $(0,1,2$ or $\geq 3 ; P=0.038)$, while the frequency of lunch and dinner was not different between the three groups after adjusting for sex (men or women), working status (full-time, part-time, housewife, student or other) and number of children (0, 1, 2 or $\geq 3)$. Furthermore, the results were the same when age was added to the adjusting factors, namely when adjusting for age, sex (men or women), working status (full-time, part-time, housewife, student or other) and number of children $(0,1$, 2 or $\geq 3$ ).

\section{Discussion}

The present study aimed to evaluate the association of breakfast skipping with nutrition knowledge level. We found that the frequency of breakfast consumption was higher in participants with high nutrition knowledge than in those with low. This finding suggests that nutrition knowledge level affects breakfast skipping among Japanese adults. To the best of our knowledge, the present study is the first not only to explore nutrition knowledge level in Japanese adults but also to examine the relationship of breakfast skipping and overall nutrition knowledge level among adults anywhere in the world.

It was reported that nutrition knowledge needs to be assessed by questionnaires with reasonable validity and reliability when assessing dietary behaviours, because it is one of the factors which influences dietary behaviours ${ }^{(28)}$. Thus, the research focusing on nutrition knowledge has been increasing. A few studies have reported that nutrition knowledge of adults assessed by a valid and reliable nutrition knowledge questionnaire is related to demographic characteristics of the participants ${ }^{(3,29-31)}$. For example, an Australian study reported that sex, age, highest level of education and employment status affected nutrition knowledge level ${ }^{(29)}$. A UK study reported that sex, educational level and socio-economic status were the factors affecting nutrition knowledge ${ }^{(31)}$. In a study on Australian military personnel, sex, age and educational background had positive relationships with nutrition knowledge ${ }^{(30)}$. Moreover, age, education level and working status were reported to affect nutrition knowledge in a study on young and middle-aged Belgian women ${ }^{(3)}$. Meanwhile, the present study found that participants' nutrition knowledge was related to the characteristics of sex, working status and number of children. Female adults had higher nutrition knowledge than male adults in the present study, similarly as previous studies ${ }^{(29-31)}$. Regarding working status, our result was slightly incoherent with previous studies ${ }^{(29,31)}$ in that full-time workers were more categorized and housewives were less categorized into the group with low nutrition knowledge level. Most housewives were women (data not shown) and women had more nutrition knowledge than men ${ }^{(29,31)}$. This might explain that housewives were less categorized into the 
Table 1 Characteristics of 1165 Japanese adults aged 18-64 years categorized into Low, Middle and High groups by nutrition knowledge level, Kanto region, Japan, January-March 2015

\begin{tabular}{|c|c|c|c|c|c|c|c|c|c|}
\hline & \multicolumn{2}{|c|}{ All (n 1165) } & \multicolumn{2}{|c|}{ Low (n 392) } & \multicolumn{2}{|c|}{ Middle ( $n$ 397) } & \multicolumn{2}{|c|}{ High (n 376) } & \multirow[b]{2}{*}{$P^{\star}$} \\
\hline & $n$ or Mean & $\%$ or SD & $n$ or Mean & $\%$ or SD & $n$ or Mean & $\%$ or SD & $n$ or Mean & $\%$ or SD & \\
\hline Sex, $n$ and $\%$ & & & & & & & & & $<0.001$ \\
\hline Men & 498 & $42 \cdot 7$ & 214 & $54 \cdot 6$ & 164 & 41.3 & 120 & 31.9 & \\
\hline Women & 667 & $57 \cdot 3$ & 178 & $45 \cdot 4$ & 233 & 58.7 & 256 & $68 \cdot 1$ & \\
\hline Age (years), mean and SD & $43 \cdot 8$ & 8.9 & $44 \cdot 1$ & 9.0 & $43 \cdot 0$ & $9 \cdot 8$ & 44.4 & $7 \cdot 8$ & 0.054 \\
\hline Education, $n$ and $\%$ & & & & & & & & & 0.093 \\
\hline University or higher & 483 & 41.5 & 158 & $40 \cdot 3$ & 154 & $38 \cdot 8$ & 171 & 45.5 & \\
\hline Junior college or vocational technical school & 352 & $30 \cdot 2$ & 109 & $27 \cdot 8$ & 128 & $32 \cdot 2$ & 115 & $30 \cdot 6$ & \\
\hline High school or junior high school & 330 & $28 \cdot 3$ & 125 & 31.9 & 115 & $29 \cdot 0$ & 90 & 23.9 & \\
\hline Annual household income, $n$ and \% & & & & & & & & & 0.053 \\
\hline$<2$ million yen & 37 & $3 \cdot 2$ & 13 & 3.3 & 13 & $3 \cdot 3$ & 11 & 2.9 & \\
\hline $2-6$ million yen & 307 & $26 \cdot 4$ & 103 & $26 \cdot 3$ & 121 & 30.5 & 83 & $22 \cdot 1$ & \\
\hline $6-10$ million yen & 490 & $42 \cdot 1$ & 177 & $45 \cdot 2$ & 158 & 39.8 & 155 & $41 \cdot 2$ & \\
\hline$>10$ million yen & 331 & 28.4 & 99 & $25 \cdot 3$ & 105 & $26 \cdot 4$ & 127 & $33 \cdot 8$ & \\
\hline Working status, $n$ and $\%$ & & & & & & & & & $<0.001$ \\
\hline Full-time & 534 & $45 \cdot 8$ & 209 & 53.3 & 177 & $44 \cdot 6$ & 148 & 39.4 & \\
\hline Part-time & 185 & $15 \cdot 9$ & 60 & $15 \cdot 3$ & 55 & 13.9 & 70 & $18 \cdot 6$ & \\
\hline Housewife & 283 & $24 \cdot 3$ & 66 & $16 \cdot 8$ & 108 & $27 \cdot 2$ & 109 & 29.0 & \\
\hline Student & 60 & $5 \cdot 2$ & 20 & $5 \cdot 1$ & 26 & 6.5 & 14 & 3.7 & \\
\hline Others & 103 & $8 \cdot 8$ & 37 & 9.4 & 31 & $7 \cdot 8$ & 35 & $9 \cdot 3$ & \\
\hline Marital status, $n$ and $\%$ & & & & & & & & & 0.157 \\
\hline Single & 122 & $10 \cdot 5$ & 35 & 8.9 & 51 & $12 \cdot 8$ & 36 & $9 \cdot 6$ & \\
\hline Married & 1043 & 89.5 & 357 & $91 \cdot 1$ & 346 & $87 \cdot 2$ & 340 & 90.4 & \\
\hline Number of children, $n$ and $\%$ & & & & & & & & & 0.002 \\
\hline 0 & 86 & 7.4 & 25 & $6 \cdot 4$ & 40 & $10 \cdot 1$ & 21 & $5 \cdot 6$ & \\
\hline 1 & 251 & 21.5 & 75 & $19 \cdot 1$ & 80 & $20 \cdot 2$ & 96 & $25 \cdot 5$ & \\
\hline 2 & 599 & 51.4 & 221 & $56 \cdot 4$ & 181 & $45 \cdot 6$ & 197 & $52 \cdot 4$ & \\
\hline$\geq 3$ & 229 & $19 \cdot 7$ & 71 & $18 \cdot 1$ & 96 & $24 \cdot 2$ & 62 & $16 \cdot 5$ & \\
\hline JGNKKQ score, mean and SD & $69 \cdot 3$ & $23 \cdot 7$ & $42 \cdot 0$ & 14.2 & $72 \cdot 3$ & 6.4 & 94.4 & $8 \cdot 7$ & $<0.001$ \\
\hline
\end{tabular}

JGNKQ, general nutrition knowledge questionnaire for Japanese adults.

${ }^{*}$ Means for continuous values were compared by ANOVA and proportions for categorical values were compared by the $x^{2}$ test between Low, Middle and High groups. 
Table 2 Difficulty of the behaviour having time to sit down and eat among 1165 Japanese adults aged 18-64 years categorized into Low, Middle and High groups by nutrition knowledge level, Kanto region, Japan, JanuaryMarch 2015

\begin{tabular}{|c|c|c|c|c|c|c|c|}
\hline & \multicolumn{2}{|c|}{ Low (n 392) } & \multicolumn{2}{|c|}{ Middle (n 397) } & \multicolumn{2}{|c|}{ High (n 376) } & \multirow[b]{2}{*}{$P^{*}$} \\
\hline & $n$ & $\%$ & $n$ & $\%$ & $n$ & $\%$ & \\
\hline Breakfast & & & & & & & 0.288 \\
\hline Very difficult & 27 & $6 \cdot 9$ & 15 & 3.8 & 14 & 3.7 & \\
\hline Difficult & 36 & 9.2 & 46 & 11.6 & 51 & 13.6 & \\
\hline In between & 28 & $7 \cdot 1$ & 31 & $7 \cdot 8$ & 22 & 5.9 & \\
\hline Easy & 62 & $15 \cdot 8$ & 64 & $16 \cdot 1$ & 61 & $16 \cdot 2$ & \\
\hline Very easy & 239 & 61.0 & 241 & 60.7 & 228 & $60 \cdot 6$ & \\
\hline Lunch & & & & & & & 0.995 \\
\hline Very difficult & 2 & 0.5 & 3 & 0.8 & 2 & 0.5 & \\
\hline Difficult & 17 & 4.3 & 17 & $4 \cdot 3$ & 16 & 4.3 & \\
\hline In between & 24 & $6 \cdot 1$ & 28 & $7 \cdot 1$ & 25 & $6 \cdot 6$ & \\
\hline Easy & 78 & 19.9 & 72 & $18 \cdot 1$ & 65 & $17 \cdot 3$ & \\
\hline Very easy & 271 & $69 \cdot 1$ & 277 & 69.8 & 268 & 71.3 & \\
\hline Dinner & & & & & & & 0.569 \\
\hline Very difficult & 5 & $1 \cdot 3$ & 2 & 0.5 & 1 & 0.3 & \\
\hline Difficult & 7 & 1.8 & 13 & 3.3 & 9 & 2.4 & \\
\hline In between & 14 & 3.6 & 17 & 4.3 & 12 & 3.2 & \\
\hline Easy & 56 & 14.3 & 66 & $16 \cdot 6$ & 58 & $15 \cdot 4$ & \\
\hline Very easy & 310 & $79 \cdot 1$ & 299 & $75 \cdot 3$ & 296 & 78.7 & \\
\hline
\end{tabular}

*Proportions for categorical values were compared by the $x^{2}$ test between Low, Middle and High groups.

Table 3 Meal frequency per week among 1165 Japanese adults aged 18-64 years categorized into Low, Middle and High groups by nutrition knowledge level, Kanto region, Japan, January-March 2015

\begin{tabular}{|c|c|c|c|c|c|c|c|c|}
\hline & \multicolumn{2}{|c|}{ Low (n 392) } & \multicolumn{2}{|c|}{ Middle ( $n$ 397) } & \multicolumn{2}{|c|}{ High (n 376) } & \multirow[b]{2}{*}{$P^{*}$} & \multirow[b]{2}{*}{$P \dagger$} \\
\hline & Mean & SE & Mean & SE & Mean & SE & & \\
\hline Breakfast & $5 \cdot 3$ & 0.1 & $5 \cdot 6$ & 0.1 & 5.7 & 0.1 & 0.038 & 0.047 \\
\hline Lunch & $6 \cdot 1$ & 0.1 & $6 \cdot 4$ & 0.1 & $6 \cdot 3$ & 0.1 & 0.064 & 0.067 \\
\hline Dinner & $6 \cdot 0$ & 0.1 & $6 \cdot 2$ & $0 \cdot 1$ & $6 \cdot 2$ & 0.1 & 0.342 & 0.370 \\
\hline
\end{tabular}

*Means for continuous values were compared by ANCOVA between Low, Middle and High groups adjusted sex (men or women), working status (full-time, part-time, housewife, student or other) and number of children $(0,1,2$ or $\geq 3$ ).

†Means for continuous values were compared by ANCOVA between Low, Middle and High groups adjusted sex (men or women), working status (full-time, part-time, housewife, student or other), number of children $(0,1,2$ or $\geq 3)$ and age.

Low group in the present study. Our study classified employed status as part-time workers separately from fulltime workers, but the Australian study categorized full-time workers and part-time workers into employed, and housewives were defined independently in our study although the Australian study categorized them into others ${ }^{(29)}$. Also, the UK study did not assess the relationship between working status and nutrition knowledge, and employment status was defined as socio-economic status combined with household income ${ }^{(31)}$. This result might be influenced because working status classification in the present study was different from other studies. However, more detailed classification of working status was used in present study, thus the relationship between nutrition knowledge and working status might reveal more than before.

Nutrition knowledge level differed according to number of children in the present study, as well as in the Australian study ${ }^{(29)}$ and the study on young and middle-aged Belgian women $^{(3)}$, in contrast to the UK study ${ }^{(31)}$. However, our results were not totally consistent with these Australian and Belgian studies. The participants' nutrition knowledge level was not influenced depending on the presence of children in the current study, while the presence of children affected the adults' nutrition knowledge level in the two previous studies $^{(3,29)}$. One possible reason for this could be that the age of participants was different between studies. The Belgian women had a median age of 30 years $^{(3)}$ and $40 \%$ of the participants were younger than 35 years old in the Australian study, ranging from 18 to 74 years ${ }^{(29)}$. Additionally, more than $20 \%$ of elderly people over 65 years old were included in a UK study ${ }^{(31)}$, while the mean age of the participants in present study was 43.8 (sD 8.9) years. Thus, these differences in the age composition of participants between studies may have produced our result that age did not affect nutrition knowledge levels unlike the other four studies $^{(3,29-31)}$.

Previous studies found that education was related to nutrition knowledge ${ }^{(3,29-31)}$, while those relationships were not seen in the present study. In the UK study, more than $40 \%$ of participants were categorized into the lowest education level $^{(31)}$, and the Belgian and Australian military personnel studies used the classification of high or low 
education $^{(3,29)}$. Only the Australian study used the same classification for education level as our study, but more than $50 \%$ of the participants were in the lowest category; completed high school or less ${ }^{(29)}$. The difference in distribution of education background might influence the difference in results between the three previous studies and the present one.

Breakfast skipping frequency among the participants in the present study was more frequent than lunch or dinner skipping, as reported in a previous study investigating breakfast, lunch and dinner frequency among US young $\operatorname{adults}^{(32)}$. However, each meal skipping frequency in the current study was less than in the previous study. It has been reported that older people have more meal frequency $^{(33)}$. In fact, the younger participants had less breakfast and lunch frequency than the older in the present study although the frequency of dinner was not different by age (data not shown). Therefore, these frequency differences might be influenced because the present study participants were older than the previous study participants.

Nutrition knowledge level was related only to breakfast skipping but not lunch and dinner skipping after adjusting for sex, working status, number of children and age in the present study. The participants who had less nutrition knowledge tended to skip breakfast frequently. This result is well consistent with the study suggesting that nutrition knowledge is necessary to improve eating behaviours ${ }^{(7)}$. A previous study also reported that participants with lower nutrition knowledge tended to miss their breakfast, although the previous study participants differed from those of the present study in terms of younger age and living in socio-economically disadvantaged neighbourhoods, and their nutrition knowledge level was measured by only part of the GNKQ ${ }^{(23)}$. Younger age and lower income were reported to have a large influence on breakfast skipping ${ }^{(27)}$. Apart from the difference in age and income status in the studies, the consistent results may indicate that nutrition knowledge is likely to be one of the factors affecting breakfast skipping.

The difference in mean breakfast frequency between High and Low groups was only $0.4 \mathrm{~d} /$ week. One reason for this relatively small difference might be that Japan has a lower rate of breakfast skipping compared with Western countries, although it is difficult to compare in exactly the same way due to differences in the definition of breakfast skipping in each study ${ }^{(11,14,15,34)}$. Even with a relatively small difference in breakfast frequency, nutrition education leading to a situation with less breakfast skipping is necessary, because it has been reported that breakfast skipping is related to low intakes of micronutrients ${ }^{(34)}$ and other unhealthy eating behaviours ${ }^{(35)}$. Therefore, the present results may suggest there is a possibility that nutrition knowledge needs to be one of the targets when conducting nutrition education.

A systematic review of studies about breakfast skipping reported that the main factors for breakfast skipping were having no time for breakfast in the morning and habituation $^{(36)}$. In the present study, the difficulty of having time to sit down and eat each meal was not significantly different according to nutrition knowledge level. Moreover, the rate of those who answered 'difficult' or 'very difficult' was low in all three nutrition knowledge groups. Thus, it is unlikely that having no time in the morning led to breakfast skipping in our study participants. Also, it has been reported that the dietary habits formed until adolescence persist throughout adult life and these behaviours can be difficult to modify later in life ${ }^{(37-41)}$. Therefore, it is impossible to deny the likelihood that the participants of the present study skipped breakfast by custom from the time of their childhood. Unfortunately, in the present study, we did not examine previous dietary habits such as those of their childhood. Further studies are needed to examine the influence of previous dietary habits on breakfast skipping in addition to nutrition knowledge.

Several limitations of the current study need to be mentioned. First, the participants in the study were not random samples from the general population and the survey area was restricted to a single region in Japan. The participants were therefore likely not representative of Japanese adults. Second, the response rate was as low as $23.6 \%$. The nutrition knowledge questionnaire used in the present study consists of ten pages, and it takes 20 to $30 \mathrm{~min}$ to answer. More than half of the participants were working, so they might not have had enough time to respond to the questionnaire. Thus, the participants might have been highly health conscious and have higher nutrition knowledge level than Japanese people. Even though the study participants were probably interested in nutrition and food, the JGNKQ scores between the three groups categorized by nutrition knowledge level were significantly different. Therefore, it may not be a problem in this research. Third, lunch was defined as consumed between 12.00 and 14.00 hours and dinner as consumed between 16.00 and 20.00 hours. If participants had lunch or dinner outside the time specified, the number of lunch or dinner was counted as zero times. Thus, there is undeniably a possibility that those factors may affect the present study results. However, Yokoyama et al. reported that dinner time was classified as regular time from 17.00 to 20.00 hours and irregular time other than that ${ }^{(27)}$. Additionally, traditional meal time periods reported are that breakfast is from 06.00 to 10.00 hours, lunch is from 12.00 to 14.00 hours and dinner is from 16.00 to 20.00 hours, although reported in Sweden ${ }^{(42)}$. All the average frequencies of lunch and dinner exceeded $6 \mathrm{~d}$ /week among participants in the present study. Thus, the definition of lunch and dinner time may not be a problem. Fourth, women with smoking habits were reported to tend to skip breakfast ${ }^{(23)}$. We did not examine the smoking status in our study. The proportion of Japanese women with smoking habits was reported as $8.2 \%$, a low percentage compared with other countries ${ }^{(20,43)}$. However, 
it is undeniable that those factors of the home environment may affect dietary behaviour. We suggest that further investigational approaches are required to clarify these uncertainties. Finally, there could be other confounding factors than those we examined. For example, some of the participants in the present study may have worked shifts. It was reported that the proportion of shift workers is about $10 \%$ in Japan ${ }^{(44)}$. However, it was impossible to distinguish about the aspect of shift workers with the item of 'working status'. Therefore, the effect on the results from such factors could not be ruled out.

\section{Conclusions}

The present cross-sectional study showed that nutrition knowledge level is related to breakfast skipping among Japanese adults. Thus, there may be a possibility that breakfast skipping has occurred because of little information about food and nutrition. Therefore, healthpromotion programmes or nutrition counselling on breakfast skipping might need to pay greater attention to nutrition knowledge level as well as dietary habits. Further studies are needed to clarify the association between breakfast skipping and nutrition knowledge and other factors related to skipping breakfast, to make a strategy for improving dietary behaviours among adults.

\section{Acknowledgements}

Financial support: This study was supported by a Grantin-Aid for Scientific Research from the Japan Society for the Promotion of Science (JSPS KAKENHI grant number 26350161). JSPS had no role in the design, analysis or writing of this article. Conflict of interest: None. Authorship: All authors contributed to the concept and design of survey and data collection. M.M., N.I. and Az.S. contributed to data management. M.M. and N.I. conducted the statistical analysis. M.M. drafted the paper. Ak.S., Az.S. and S.I. provided input for the final draft of the manuscript. All authors read and approved the final manuscript. Ethics of buman subject participation: This study was conducted according to the guidelines laid down in the Declaration of Helsinki and all procedures involving human subjects were approved by the Ethics Committee of Seitoku University (approval number H26U035). Written informed consent was obtained from all subjects.

\section{References}

1. Birch LL \& Davison KK (2001) Family environmental factors influencing the developing behavioral controls of food intake and childhood overweight. Pediatr Clin North Am 48, 893-907.
2. Wardle J, Parmenter K \& Waller J (2000) Nutrition knowledge and food intake. Appetite 34, 269-275.

3. De Vriendt T, Matthys C, Verbeke W et al. (2009) Determinants of nutrition knowledge in young and middle-aged Belgian women and the association with their dietary behaviour. Appetite 52, 788-792.

4. Ball K, Crawford D \& Mishra G (2006) Socio-economic inequalities in women's fruit and vegetable intakes: a multilevel study of individual, social and environmental mediators. Public Health Nutr 9, 623-630.

5. Dallongeville J, Marecaux N, Cottel D et al. (2001) Association between nutrition knowledge and nutritional intake in middle-aged men from Northern France. Public Health Nutr 4, 27-33.

6. Dickson-Spillmann M \& Siegrist M (2011) Consumers' knowledge of healthy diets and its correlation with dietary behaviour. J Hum Nutr Diet 24, 54-60.

7. Worsley A (2002) Nutrition knowledge and food consumption: can nutrition knowledge change food behaviour? Asia Pac J Clin Nutr 11, Suppl. 3, S579-S585.

8. Levy AS, Fein SB \& Stephenson M (1993) Nutrition knowledge levels about dietary fats and cholesterol: 1983-1988. J Nutr Educ 25, 60-66.

9. Deshmukh-Taskar PR, Radcliffe JD, Liu Y et al. (2010) Do breakfast skipping and breakfast type affect energy intake, nutrient intake, nutrient adequacy, and diet quality in young adults? NHANES 1999-2002. J Am Coll Nutr 29, 407-418.

10. Chen J, Cheng J, Liu Y et al. (2014) Associations between breakfast eating habits and health-promoting lifestyle, suboptimal health status in Southern China: a population based, cross sectional study. J Transl Med 12, 348.

11. Zhang L, Cordeiro LS, Liu J et al. (2017) The association between breakfast skipping and body weight, nutrient intake, and metabolic measures among participants with metabolic syndrome. Nutrients $\mathbf{9}$, E384.

12. Song WO, Chun OK, Obayashi S et al. (2005) Is consumption of breakfast associated with body mass index in US adults? J Am Diet Assoc 105, 1373-1382.

13. van der Heijden AA, Hu FB, Rimm EB et al. (2007) A prospective study of breakfast consumption and weight gain among US men. Obesity (Silver Spring) 15, 2463-2469.

14. Watanabe Y, Saito I, Henmi I et al. (2014) Skipping breakfast is correlated with obesity. J Rural Med 9, 51-58.

15. Sakurai M, Yoshita K, Nakamura K et al. (2017) Skipping breakfast and 5-year changes in body mass index and waist circumference in Japanese men and women. Obes Sci Pract 3, 162-170.

16. Stote KS, Baer DJ, Spears K et al. (2007) A controlled trial of reduced meal frequency without caloric restriction in healthy, normal-weight, middle-aged adults. Am J Clin Nutr $\mathbf{8 5}$, 981-988.

17. Shafiee G, Kelishadi R, Qorbani M et al. (2013) Association of breakfast intake with cardiometabolic risk factors. $J$ Pediatr (Rio J) 89, 575-582.

18. Farshchi HR, Taylor MA \& Macdonald IA (2005) Deleterious effects of omitting breakfast on insulin sensitivity and fasting lipid profiles in healthy lean women. Am J Clin Nutr 81, 388-396.

19. Cahill LE, Chiuve SE, Mekary RA et al. (2013) Prospective study of breakfast eating and incident coronary heart disease in a cohort of male US health professionals. Circulation 128, 337-343.

20. Ministry of Health, Labour and Welfare (2017) The National Health and Nutrition Survey in Japan, 2016. http://www.mhlw.go.jp/bunya/kenkou/eiyou/h28-houkoku. html (accessed April 2018).

21. Kant AK \& Graubard BI (2015) 40-year trends in meal and snack eating behaviors of American adults. J Acad Nutr Diet 115, 50-63. 
22. Reeves S, Halsey LG, McMeel Y et al. (2013) Breakfast habits, beliefs and measures of health and wellbeing in a nationally representative UK sample. Appetite 60, 51-57.

23. Smith KJ, McNaughton SA, Cleland VJ et al. (2013) Health, behavioral, cognitive, and social correlates of breakfast skipping among women living in socioeconomically disadvantaged neighborhoods. J Nutr 143, 1774-1784.

24. Parmenter K \& Wardle J (1999) Development of a general nutrition knowledge questionnaire for adults. Eur J Clin Nutr 53, 298-308.

25. Matsumoto M, Tanaka R \& Ikemoto S (2017) Validity and reliability of a general nutrition knowledge questionnaire for Japanese adults. J Nutr Sci Vitaminol (Tokyo) 63, 298-305.

26. Timlin MT \& Pereira MA (2007) Breakfast frequency and quality in the etiology of adult obesity and chronic diseases. Nutr Rev 65, 268-281.

27. Yokoyama Y, Onishi K, Hosoda T et al. (2016) Skipping breakfast and risk of mortality from cancer, circulatory diseases and all causes: findings from the Japan Collaborative Cohort Study. Yonago Acta Med 59, 55-60.

28. Spronk I, Kullen C, Burdon C et al. (2014) Relationship between nutrition knowledge and dietary intake. Br J Nutr 111, 1713-1726.

29. Hendrie GA, Coveney J \& Cox D (2008) Exploring nutrition knowledge and the demographic variation in knowledge levels in an Australian community sample. Public Health Nutr 11, 1365-1371.

30. Kullen CJ, Iredale L, Prvan T et al. (2015) Evaluation of general nutrition knowledge in Australian military personnel. J Acad Nutr Diet 116, 251-258.

31. Parmenter K, Waller J \& Wardle J (2000) Demographic variation in nutrition knowledge in England. Health Educ Res 15, 163-174.

32. Nelson Laska M, Larson NI, Neumark-Sztainer D et al. (2010) Dietary patterns and home food availability during emerging adulthood: do they differ by living situation? Public Health Nutr 13, 222-228.

33. Jung CH, Lee JS, Ahn HJ et al. (2017) Association of meal frequency with metabolic syndrome in Korean adults: from the Korea National Health and Nutrition Examination Survey (KNHANES). Diabetol Metab Syndr 9, 77.
34. Leech RM, Livingstone KM, Worsley A et al. (2016) Meal frequency but not snack frequency is associated with micronutrient intakes and overall diet quality in Australian men and women. J Nutr 146, 2027-2034.

35. Huang C, Momma H, Cui Y et al. (2017) Independent and combined relationship of habitual unhealthy eating behaviors with depressive symptoms: A prospective study. $J$ Epidemiol 27, 42-47.

36. Pendergast FJ, Livingstone KM, Worsley A et al. (2016) Correlates of meal skipping in young adults: a systematic review. Int J Behav Nutr Phys Act 13, 125.

37. St-Onge MP, Keller KL \& Heymsfield SB (2003) Changes in childhood food consumption patterns: a cause for concern in light of increasing body weights. Am J Clin Nutr 78, 1068-1073.

38. Campbell KJ \& Hesketh KD (2007) Strategies which aim to positively impact on weight, physical activity, diet and sedentary behaviours in children from zero to five years. A systematic review of the literature. Obes Rev $\mathbf{8}$, 327-338.

39. Mikkila V, Rasanen L, Raitakari OT et al. (2005) Consistent dietary patterns identified from childhood to adulthood: the Cardiovascular Risk in Young Finns Study. Br J Nutr 93, 923-931.

40. Kelder SH, Perry CL, Klepp KI et al. (1994) Longitudinal tracking of adolescent smoking, physical activity, and food choice behaviors. Am J Public Health 84, 1121-1126.

41. Guo SS, Wu W, Chumlea WC et al. (2002) Predicting overweight and obesity in adulthood from body mass index values in childhood and adolescence. Am J Clin Nutr 76, 653-658.

42. Berteus Forslund H, Lindroos AK, Sjostrom L et al. (2002) Meal patterns and obesity in Swedish women - a simple instrument describing usual meal types, frequency and temporal distribution. Eur J Clin Nutr 56, 740-747.

43. World Health Organization (2016) World Health Statistics 2016: Monitoring health for the SDGs. http://www.who.int/ gho/publications/world_health_statistics/2016/en/ (accessed April 2018).

44. Kubo T (2014) Estimate of the number of night shift workers in Japan. J UOEH 36, 273-276. 